\title{
Design of a versatile Half-Bridge Converter Able to Drive 6x6, 6x4, 8x6, 12x8 Switched Reluctance Generators and Motors Using Arduino
}

\author{
R. J. Dias ${ }^{1}$, D. G. Pereira ${ }^{1}$, R. N. Guimarães ${ }^{1}$, A. S. Nogueira ${ }^{1}$ and L.L. Silva ${ }^{1}$ \\ ${ }^{1}$ Federal Institute of Education, Science and Technology of Goiás \\ Campus of Goiânia - Goiás, (Brazil) \\ e-mail: renatojayme.ee@gmail.com, gdeborah43@gmail.com, eng.rodrigo.ng@gmail.com
}

\begin{abstract}
This paper presents the studies required for the design and construction of a versatile Half-Bridge converter. This converter can drive a wide variety of machines - from one to four phases. The converter was designed mainly to drive Variable Reluctance Machines in $6 \times 4,6 \times 6,8 \times 6$ and $12 \times 8$ topologies, working as both motor and generator. The versatility of the proposed converter is its main advantage, as in both academic and industrial applications, this feature can generate financial and production time savings. An analysis was carried out among the main converter topologies to justify the choice of the Half-Bridge topology followed by a project detailing of the chosen converter. For the design and construction of this converter, only commercially available devices were considered. To control the triggering keys, the Arduino platform was used due to its easy access and low cost. After the converter power circuit definition, the circuit for communication between the sensor (encoder), the controller (Arduino), and the switches (IGBT) is presented, and the project assembly is shown at the end.
\end{abstract}

Keywords. Design, Half-Bridge Converter, Versatility, Switched Reluctance Machine, Arduino.

\section{Introduction}

The Switched Reluctance Machine (SRM) is an electromechanical energy converter that uses direct current excitation to drive its coils. It consists of a doubleprojecting laminate structure that is constructively simple, cost-effective and robust [1]. It was one of the first electric machines manufactured around 1838 [2]. It presents nonlinear electromagnetic behaviour, torque ripple, and the need for electronic switching [9]. The study of the use of these machines has proven to be advantageous in recent decades due to constant developments in semiconductor technology. Currently, SRM research is focusing primarily on activities that require variable speed. Aeronautics, hybrid cars and wind power are the main areas of interest [3] [4] [10] [11]. One of the main steps for SRM operation is to switch the excitation of its coils correctly. This is done with the converter. In this paper, a generic converter is introduced as a versatile tool for the use of many of the mentioned applications.

\section{SRM Operation Principles}

Figure 1 shows the cross-section of a $6 \times 4$ SRM, i.e. it has 8 poles on the stator and 6 on the rotor. The windings of one of the phases (A) are shown. The machine has four phases (each pair of poles in the stator constitutes one phase). Phase A of this machine is completely aligned with the rotor, which corresponds to a position of maximum inductance.

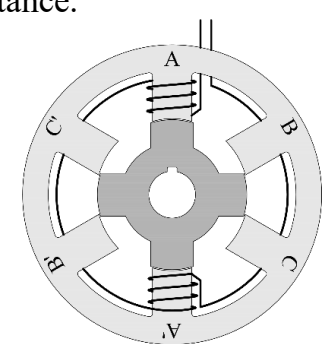

Fig.1. 8x6 SRM showing phase A winding.

To drive SRM as a motor, the corresponding coil must be energized while the inductance variation is positive (1).

$$
T_{\text {emg }}=\frac{1}{2} i^{2} \frac{d L(i, \theta)}{d \theta} \geq 0
$$

Driving the machine as a generator requires that the corresponding coil is energized while the inductance variation is negative (2).

$$
T_{\text {emg }}=\frac{1}{2} i^{2} \frac{d L(i, \theta)}{d \theta} \leq 0
$$

That is, the direction of the conjugate ( $\left.T_{\text {eng }}\right)$ depends on the derivative of the inductance $(d(\theta) / d \theta)$ [5], being necessary to know the angular position of the rotor at all times. Switching affects the efficiency and performance of the machine as it defines the pulses in the inductance profile of each phase. Therefore, one of the main steps for SRM operation is to correctly switch the excitation of the stator coils. This specific task is transferred to the converter. In SRM, each coil is controlled independently. Thus, each coil is always connected in series with at least one key. To determine the angular position of the rotor in relation to the phase, an optical sensor (encoder) is implanted next to the rotor of the machine. This signal is processed with the aid of a microprocessor (Arduino), 
which then sends command signals for switching the buttons contained in the converter.

\section{Choice of Converter Topology}

This paper assumes the drive of Variable Reluctance Machines with powers up to $1 \mathrm{hp}$, either motor or generator, in $6 \times 6,6 \times 4,8 \times 6$ and $12 \times 8$ topologies. Therefore, the converter must be robust, efficient and also versatile. From these requirements, a comparative analysis was made between four of the main converter topologies: Half-Bridge (HB), C-Dump, $\mathrm{N}+1$ and $1.5 \mathrm{~N}$.

The C-Dump was first introduced by [13] in 1987. This topology is characterized by using only one key to energize each phase. Despite being a robust converter, it has a complex logical sequence of coil switching and limited use in high-speed applications [6];

In the $\mathrm{N}+1$ topology, $\mathrm{N}+1$ keys are used, one key for each phase and one shared key. Because it has a diode and a switch that will be shared by the other coils, independent control of the coils is impossible [6];

In the $1.5 \mathrm{~N}$ topology, each two phases require three switches and three diodes for the drive, presenting itself as an evolution of $\mathrm{N}+1$, since it enables independent control of the coils. However, its constitution limits the machine topologies that can be driven by this converter, which must have an even number of phases, not being compatible with SRM with odd phase numbers [6].

The Half-Bridge (HB) converter uses two switches and two diodes to drive each phase - which increases its manufacturing cost. On the other hand, it features simpler coil switching logic sequence, independent phase control, high fault tolerance and, above all, the ability to drive a wide variety of machines [6]. Thus, its great versatility and high-performance features make this topology the most cost-effective.

\section{A. Conversor Half-Bridge (HB)}

Due to its versatility and other advantages mentioned above, the Half-Bridge topology was chosen for the design and construction of a converter. An HB converter arm for single-phase drive is shown in Fig. 2 (a). The switches represented by $\mathrm{c} 1$ and $\mathrm{C} 1$ may consist of Transistors, Metal-Oxide-Semiconductor Field Effect Transistors (MOSFETs), and Insulated Gate Bipolar Transistor (IGBTs). The converter contains a drive circuit for each phase of the machine. The Half-Bridge (HB) converter has an operation elassified divided into four stages: excitation, freewheel, regeneration and deactivation (Fig. 2).

The excitation stage (Fig. 2 (b)) begins when the encoder identifies a preset angular position, and through the microcontroller (Arduino), sends commands to switches $\mathrm{cl}$ and $\mathrm{C} 1$. These switches are closed at the same time and the machine's coil (Phase 1) is supplied with direct current (VE). There is then current circulation in SRM Phase 1 (I1).
When the angular position in Phase 1 reaches a preset value, one of the keys $\mathrm{c} 1$ or $\mathrm{C} 1$ will open, allowing to cancel the voltage applied to the phase and also to decrease or limit the value of the current in the coil. The current flows from the coil through the closed switch and the directly polarized diode, partially demagnetizing the phase, limiting the current in the SRM phase, and conveniently switching $\mathrm{cl}$ or $\mathrm{C} 1$. This current variation (within the established lower and upper limits) may last as long as there is a positive inductance variation in phase [7]. This stage is called freewheel (Fig. 2 (c)).

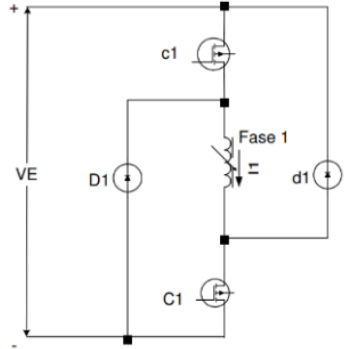

(a)

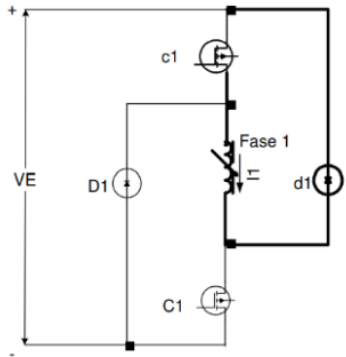

(c)

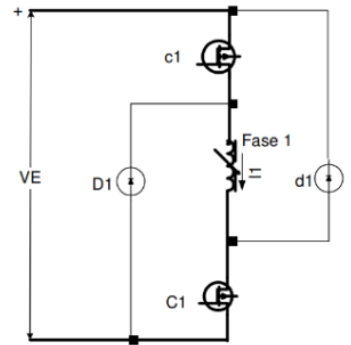

(b)

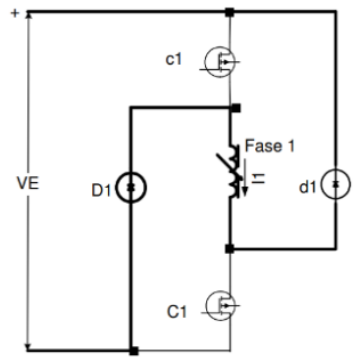

(d)
Fig.2. (a) HB converter arm; (b) Active circuit in the excitation stage; (c) Active circuit in freewheel stage; (d) Active circuit in regeneration step for reluctance motor [7].

The regeneration phase (Figure 2 (d)) begins when the encoder re-identifies a predefined angular position and sends commands through the Arduino to switches $\mathrm{cl}$ and C1 to open simultaneously, thereby blocking the current flow provided by the source (VE) in Phase 1. When switches $\mathrm{c} 1$ and $\mathrm{C} 1$ are open, the energy stored in the magnetic field (hysteresis) in phase 1 causes the current (I1) to remain in the same direction until it dissipates. Therefore, the diodes $\mathrm{d} 1$ and $\mathrm{d} 1$ become the only possible path for current flow. This energy can be reused for the next phase excitation (when used as a motor) or for feeding a load (when used as a generator). The regeneration must be stopped before the inductance variation becomes negative. Therefore, the production of SRM-negative conjugates is avoided unless it is desired for speed control.

The regeneration stage is important for the good performance of a reluctance motor, as the energy spent on phase excitation is high when compared to the energy used for energy conversion. Restoring this energy reflects an increase in engine efficiency [7]. In the regeneration stage, the current (I1) is cancelled, and from that point the coil remains deactivated, during which the inductance remains negative. This stage is called deactivation. The 
coil will be excited again when the inductance is positive again, restarting the cycle.

The HB converter is widely used [6] [12] because, in addition to its advantages, it allows the regeneration stage when needed, even if its circuit is slightly complex. This topology also presents a few differences in the electrical connection for SRM drives, such as MR and GR. The strategies to perform the regeneration step in MR and the generation of electricity in GR are points of greatest distinction between these two applications. Figure 3 shows a solution proposed in [8] to unify the two topologies.

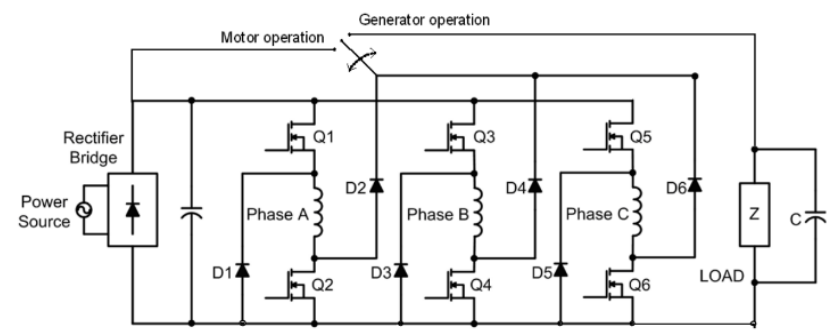

Fig.3. HB converter with a key for switching between MR and GR [8].

The key in the upper corner (Fig. 3) has the function of switching the converter between the topologies for MR and GR. With the switch closing the circuit on the right, there is the generator topology, because the cathodes of diodes D2, D4 and D6 are connected to the load. The situation in which the switch is closed on the left has the motor topology, where the cathodes of diodes D2, D4 and D6 are connected to the source.

Among the proposed machines, there are a single-phase (6x6), two-phase (6x4 and $12 \times 8)$ and a four-phase $(8 \times 6)$ machine. Therefore, the constructed converter must have 4 arms to operate machines with up to 4 phases. A viable commercial solution was sought to construct the converter, and the most economical option found was an IGBT module (7MBR50UA120), typically found in frequency converters consisting of 7 IGBT switches. Its construction prevents a coil from being connected in series between the keys. Thus, only half of the keys are used in each module and therefore require more IGBT modules. In addition, in order to mount the circuit according to the HB converter model of Figure 3, each of the switches must be individually tripped and electrically isolated from each other [7]. The following converter configuration was proposed, shown in Figure 5. Since 3 key pairs can be used in a module, a 3-phase machine would require 2 IGBT modules for its converter. For a four-phase SRM, it would take at least 8 IGBT switches or 4 modules.

For phases A, B and C, the gates of the bottom switches (with gates on terminals 13,12 and 11) of module number 1 are shorted to the return of the control circuit (terminal 10) (Figure 5), as a way to ensure that there is no potential difference between emitter and gate. Therefore, these switches always remain open and electrically isolate the upper switches (with ports on terminals 20, 18 and 16). In module 3, the gate and emitter terminals of the top switches are also shorted, isolating the bottom switches. Switches with shorted gate and emitter connections are always open, so only the diodes to the right of these switches are available for operation.

In order to save the IGBT switches of the trip circuit, it was decided not to use the $\mathrm{AC} / \mathrm{DC}$ converter rectifier already integrated into the IGBT modules 7MBR50UA120, which is why the three-phase rectifier SQL-50A (left in Fig. 5) was used to convert the threephase AC voltage in DC voltage. The diodes $(\mathrm{d} 1, \mathrm{~d} 2, \mathrm{~d} 3$, and $\mathrm{d} 4)$ connected to terminals $21(+)$ and $23(-)$ of the SQL 50A rectifier and the two capacitors $(\mathrm{C} 1$ and $\mathrm{C} 2)$ connected to the load and source have been added externally.

Since there would be unused keys, it was decided to use in the fourth module two of the upper keys (with ports at terminals 18 and 16) with the function of switching between the motor (MR) and generator (GR) topologies. Therefore, at the respective lower switches (with a gate at terminals 12 and 11), their gates were also shorted to the return of the control circuit (terminal 10). If the switch (gate on terminal 18) is closed and (gate on terminal 18) open, it has the topology for MR and doing the reverse, the topology for GR. These keys remain interlocked in Arduino programming for the converter. In SRM, however, the regeneration stage in the motor and generator topology changes as both MR and GR phase excitations occur equally. Figures 6 and 7 show the active circuit in the regeneration of each one.

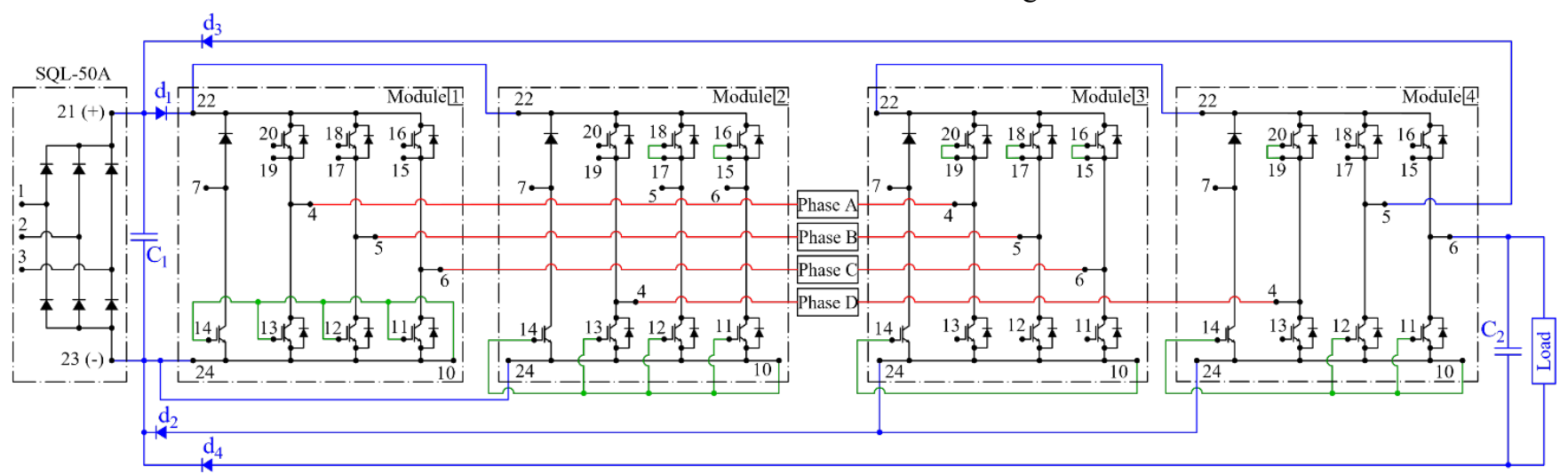

Fig.5. Four-Arm HB Converter General Diagram. 


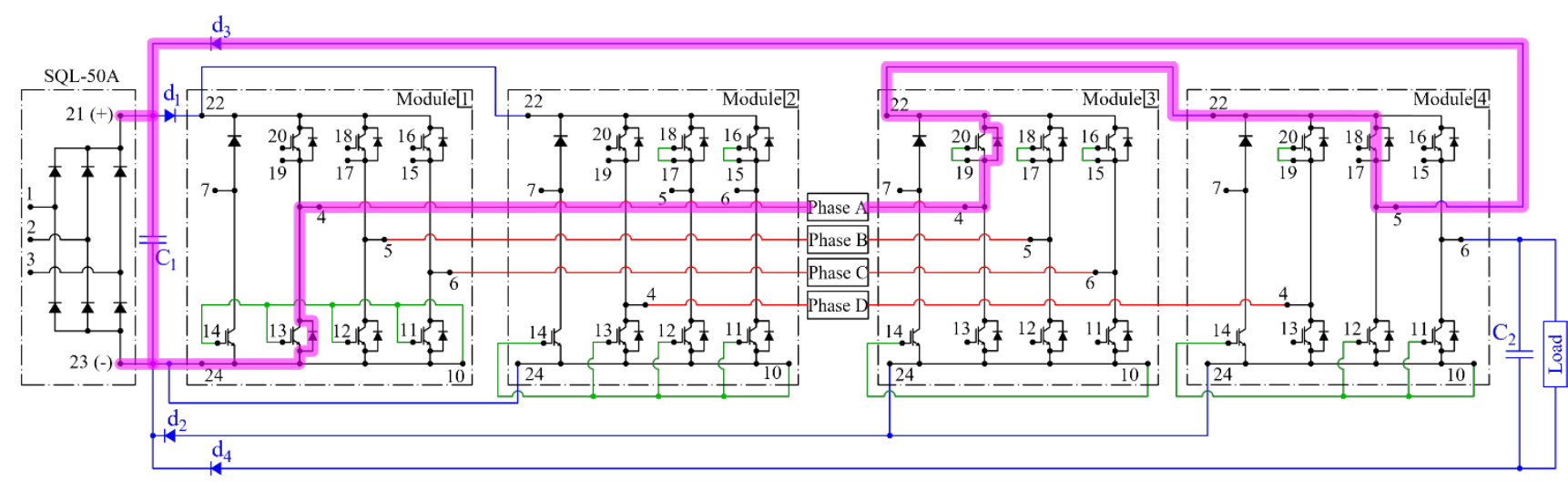

Fig.6. Active circuit in the Regeneration step for Reluctance Motor.

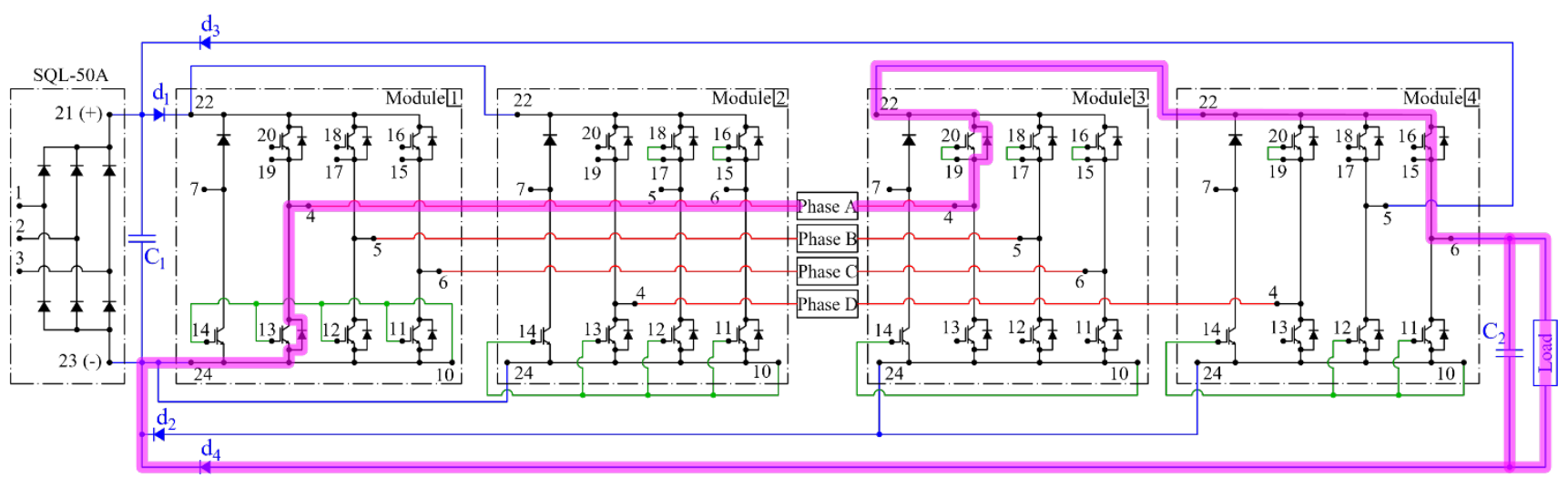

Fig.7. Active circuit in the Regeneration step for Reluctance Generator.

\section{Driver Circuit to HB Converter}

\section{A. Position Sensor (Encoder)}

The encoder is a signal transducer whose function is to detect the rotor angular position with respect to the stator, digitize that information, and send it to the microcontroller (Arduino). For this purpose, the axis of this device is coupled to the machine axis. It was used a 10-bit Gray coded absolute encoder that operates at 15 VDC. The 10bit encoder reads 1024 positions for one revolution. The Gray code is characterized by having only 1 bit changed in each transition. This feature reduces the risk of adjacentbit read errors and makes it widely used for rotating machine applications as well as the reason for its use in this work. The coder, if it is absolute, refers to the fact that its count is not cumulative, that is, the angular position sent to the Arduino is the real and instantaneous position.

\section{B. Programming Interface (Arduino)}

The Arduino microprocessor was used due easy access, low cost and simple language. It shall execute the programming logic that allows it to send the sequential drive commands of the variable reluctance machine coils. First, however, the rotor position data must be calculated, which informs the encoder. The outputs of the 10 electrical encoder signals are input to the digital readout ports of the Arduino. The encoder signals are in the range of $15 \mathrm{~V}$, but Arduino supports a voltage range of 3.3 to $5 \mathrm{~V}$. Therefore, a voltage divider circuit had to be implemented, which corresponds to the permissible voltage range. For this circuit, two resistors (12 kilohms and 3.9 kilohms) were used for each of the 10 signal cables coming from the encoder.

After reading the information position in Arduino, the encoding transformation is performed from Gray to binary. To convert from Gray to binary, programming logic is implemented to perform an or-exclusive (XOR) operation on its previous bit. Then, the transformation is performed from binary to decimal and finally from decimal to degree. If the angular position information is available in degrees at a particular time, the phases may be triggered by sending the command signals from a logic programming.

Each Reluctance Machine topology has an angular drive position range for better performance. These angle values may depend on factors, such as: number of poles in rotor and stator; application (motor or generator); inductance profile obtained with simulations; and also from empirical corrections, by previous experimental results. However, it was not the focus of this work to determine these angles, but the design and construction of a converter to drive it, and therefore generic angles were used.

The implemented programming logic sends command signals. The coil of one of the phases A, B, C and/or D will be excited when the position of the rotor angle $(\alpha)$ relative to the stator of the phase in question is within one of the specified ranges. If the angular position is outside of these ranges, the command signals will remain at the LOW logic level, thereby opening the IGBT switches of 


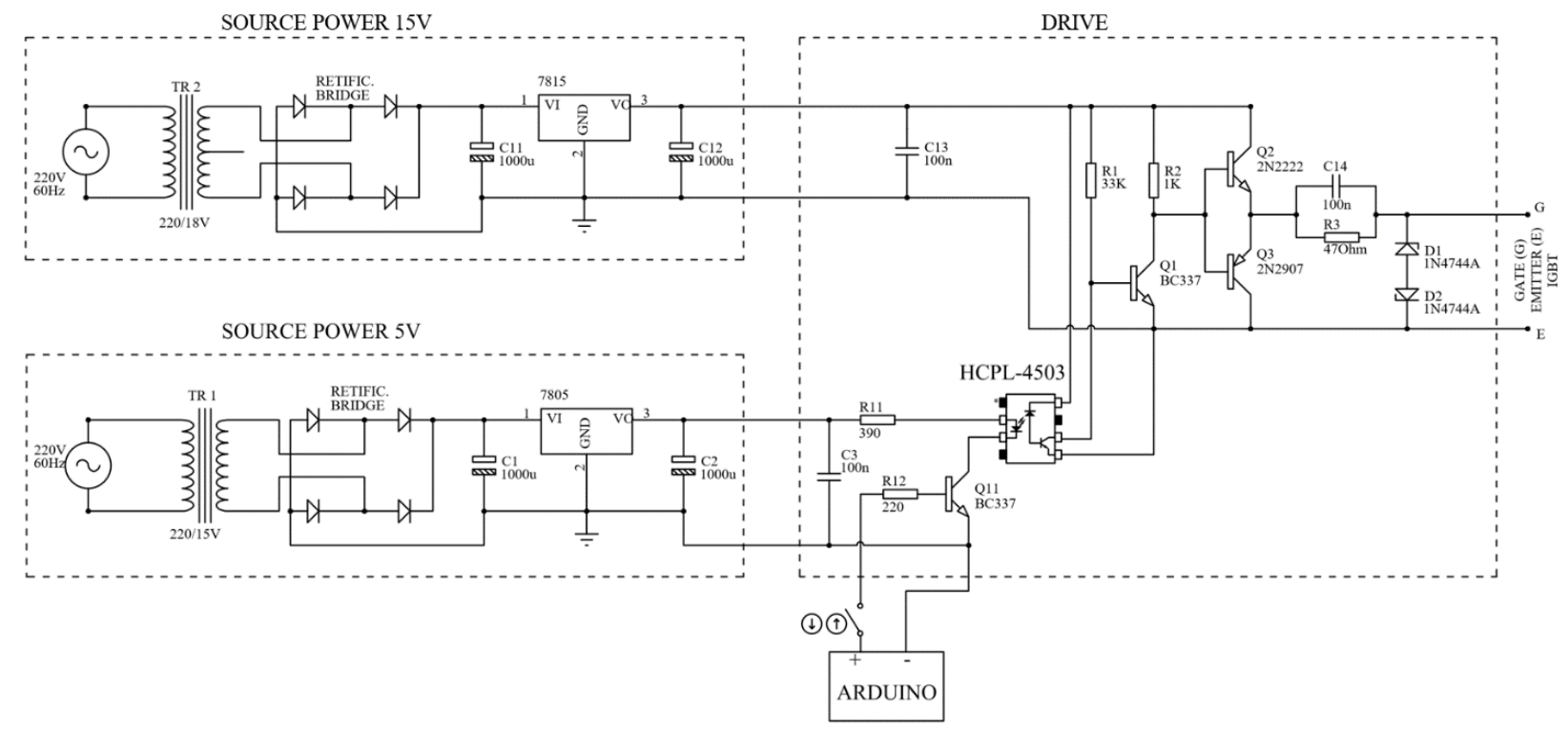

Fig.8. Driver Circuit General Diagram.

the converter and thus de-energizing the coils of the respective phases.

\section{Drive Circuit to HB Converter}

The sequential activation of machine phases at the right times requires of a microprocessor (Arduino). It can be seen that the gate voltage for driving the IGBT switches in the converter is in the range of approx. $15 \mathrm{~V}$. However, the microprocessors provide only an output voltage of $3.3 \mathrm{~V}$ or $5 \mathrm{~V}$, which is insufficient to polarize the gate and emitter of the switches used in the HB converter. Therefore, a circuit is required for coupling between systems operating at different voltage levels, commonly referred to as a driver circuit. Fig. 8 shows the proposed circuit for coupling between systems so that the command signals can reach the IGBT switches with a voltage of $15 \mathrm{~V}$. A Driver circuit is employed in situations where a control circuit does not have sufficient power to drive a switch directly. There are several ways to build a driver circuit, and the circuit used in this work was an HCPL-4503 optical coupler that couples the system when operating on different voltages in the same circuit.

Both sources, $15 \mathrm{~V}$ and $5 \mathrm{~V}$, are structurally similar. At the output of the $5 \mathrm{~V}$ source, another capacitor is connected for unwanted frequency filter, $\mathrm{C} 3$, and a resistor, R11, which is connected to pin 2 of the HCPL-4503. Pin 3 of the HPCL-4503 is connected to the collector of transistor Q11, whose Arduino outputs are connected to the base and emitter terminals. Thus, when the Arduino output is high, there is a Q11 bias, allowing current to flow between pins 2 and 3 of the HCPL-4503. Within the optocoupler, the photosensitive internal transistor passes current and thus polarizes the terminals of transistor Q1 in the $15 \mathrm{~V}$ circuit. Resistors R1 and R2 cause a voltage drop in the bases of Q1, Q2 and Q3, thus avoiding a short in the $15 \mathrm{~V}$ supply circuit. Current flows between the resistor R3 and the capacitor $\mathrm{C} 14$ to the $\mathrm{RC}$ filter, and the Zener diodes D1 and $\mathrm{D} 2$ provide a voltage level of $15 \mathrm{~V}$ at the output of the terminal at each IGBT switch.
In Fig. 5, it can be observed that the lower keys of the IGBT have one point in common. This means that only a $15 \mathrm{~V}$ circuit is required to power all of these switches. While the top switches are isolated from each other, each requires a $15 \mathrm{~V}$ circuit to power them. Command signals coming from Arduino require low power and therefore only a $5 \mathrm{~V}$ source circuit is sufficient to power all Arduino command outputs. From the scheme shown in Figure 11, a printed circuit board (PCB) has been developed to execute the drive circuit.

\section{Construction and Bench Tests}

In the design and dimensioning of cables and other materials used, the voltage and current levels of the various machines that this converter can drive had to be observed. Finally, from the compilation of all designed circuits presented in Figures 5 and 8, the converter was built, as shown in Figure 9.

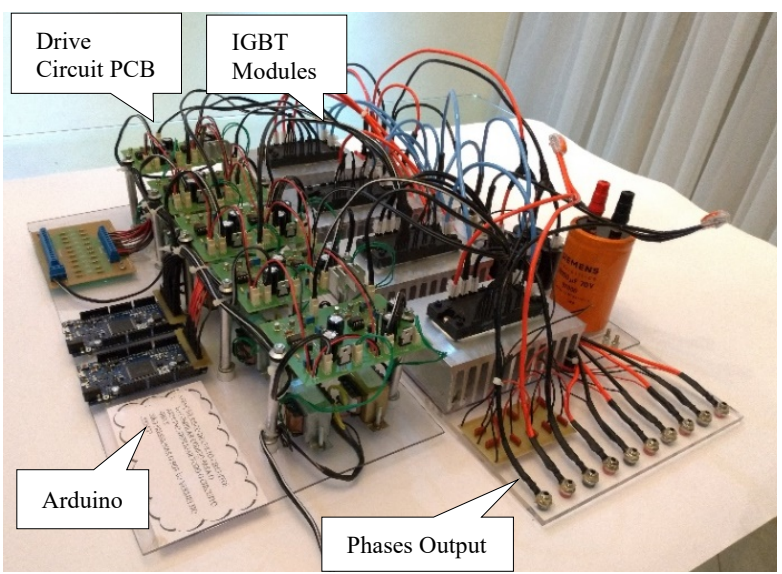

Fig.9. Converter assembly.

After the converter construction, it was submitted to the test bench to check whether the switching was being performed correctly. For this purpose, the test results are presented with the electrical tests carried out in the 
converter on the test bench. The built-in converter triggered an existing SRM with $6 \times 4$ topology in the laboratory, which acted as a generator and then as a motor. The system was operated with a sufficient minimum voltage to observe the Voltage (Channel 1, in yellow) and Current (Channel 2, in blue) curves in Phase A of this machine, and thus the switching. The results for generator (Figure 10) and motor (Figure 11) obtained with the oscilloscope are shown below.

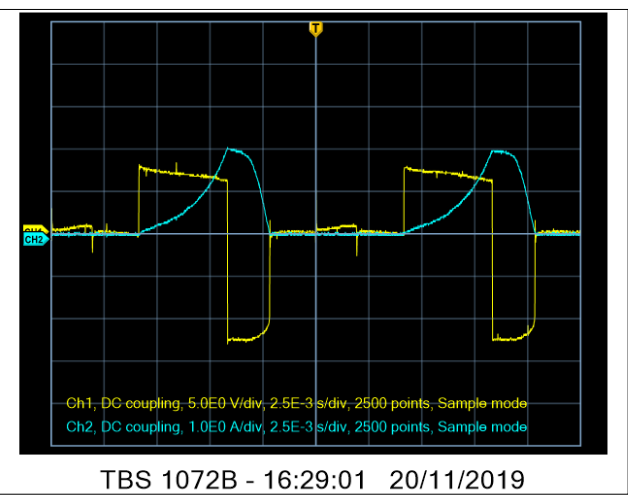

Fig.10. Voltage and Current in phase A for $6 \times 4$ SRM Generator.

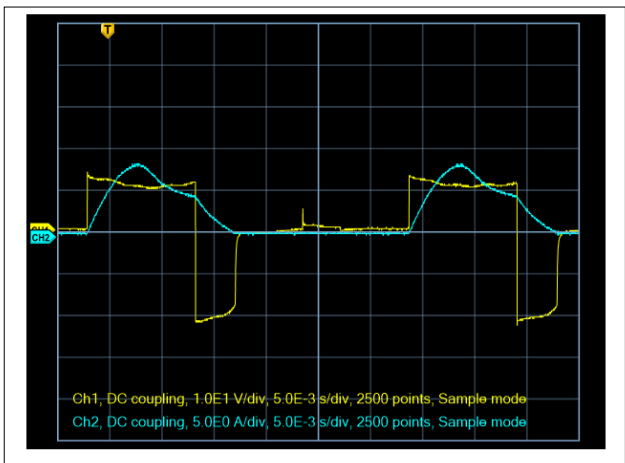

TBS 1072B - 19:20:36 03/12/2019

Fig.11. Voltage and Current in phase A for $6 \times 4$ SRM Motor.

The electrical tests on SRM 6x4 showed expected and typical behaviour, and were satisfactory, so that the correct switching of the built converter could be observed.

\section{Conclusion}

Understanding the operation of the designed circuit required analysis on all possible current paths. Through these analyses, have reached the design of the circuits specified in Figures 6 and 11. For the construction, it was observed the voltage and current levels that this converter can be submitted, given the various machines that it can drive, resulting in the built circuit (Fig. 12 and 13). In addition, its command is executed through the Arduino platform, known for its simplicity and easy access. The built-in converter was tested and is considered ready for operation of the many applications specified. The designed converter opens up countless possibilities: for academic applications, it is very cost-effective, since a single converter allows to drive a wide variety of machines, as well as the study of the control of these machines. For industrial applications, whether in applications such as motor or generator, the converter is robust and has high fault tolerance and can be used in both wind power generation and hybrid car technologies. It is evident, therefore, that the versatility of the converter proposed here is its main feature, as it can drive switched reluctance machines with single-phase or polyphase of up to four phases, both as motor and generator.

\section{Acknowledgement}

The authors thank Sanjay N. Babulal for their contributions in the text.

\section{References}

[1] R, Krishnan. Switched Reluctance Motor Drives: Modeling, Simulation, Analysis, Design and Applications. (Industrial Electronic Series), Boca Raton, CRC Press (2001).

[2] T.J.E, Miller, Electronic Control of Switched Reluctance Machines. Oxford: Newnes, (2001). xiv, 272 p.

[3] M.B.S. Pinto, G.P. Viajante, M.E. Oliveira, N. Chaves and M.A.A. Freitas, "Study and Implementation of a Computational Platform to Drive a Switched Reluctance Motor 8x6 for Electric Vehicles Applications". ICREPQ, Tenerife (Spain), ISSN 2172038 X, No.17 July 2019.

[4] S. M. Alagab, S.B. Tennakoon and C.A. Gould. "A Compact DC-DC Converter for Offshore Wind Farm Application". Renewable Energy and Power Quality Journal (RE\&PQJ) ISSN 2172-038 X, No.15 April 2017.

[5] M. V., de Paula, T. A. S. Barros, P. J. Santos Neto, R. R. de Souza, and E. Ruppert Filho, "Comparative study of torque ripple minimization techniques for a three-phase switched reluctance motor", Renewable Energy and Power Quality Journal (RE\&PQJ-2018). Vol. 1, pp. 270-275.

[6] M.J. Guan, C. Liu, K. Wang, C. Zhou. "Comparative Research of Power Converters for Multi-phase Switched Reluctance Motor". Nanjing University of Aeronautics and Astronautics, No.29 Jiangjun Road, Jiangning District, Nanjing, China. IEEE (2016)

[7] A. V. F, Silveira. "Modelagem, Construção, Testes e Análise de Desempenho de um Gerador a Relutância Chaveado". Federal University of Uberlândia, abril, 2008.

[8] A. W. F. V. Silveira, D. A. Andrade, L. C. Gomes, C. A. Bissochi JR., V. R. Bernardeli, A. Fleury. "Estratégia de Controle para o GRV Baseada na Variação do Ângulo de Magnetização". Federal University of Uberlândia, (2012).

[9] R., Abdel-Fadil, L. Számel. "State of the Art Of Switched Reluctance Motor Drives and Control Techniques". Twentieth International Middle East Power Systems Conference (MEPCON), 2018.

[10] K. M. Rahman, B. Fahimi, G. Suresh, A. V. Rajarathnam, M. Ehsani. "Advantages of Switched Reluctance Motor Applications to EV And HEV: Design And Control Issues". IEEE Trans. on Industry Applications, vol. 36, no. 1, pp. 111$121,2000$.

[11] H. Chen, Z. Yang, H. Cheng. "Average Torque Control of Switched Reluctance Machine Drives for Electric Vehicles". IET Electric Power Applications, vol. 9, pp. 459-468, 2015.

[12] S. Woothipatanapan, A. Jangwanitlert, P. Chancharoensook. "Efficiency Improvement of Converter for Switched Reluctance Motor Drives by Mixed Parallel Operation of IGBT and MOSFET". TENCON 2010 - 2010 IEEE Region 10 Conference, 2010.

[13] M. Ehsani, J. T. Bass, T. J. E. Miller, R. L. Steigerwald. "Development of a Unipolar Converter for Variable Reluctance Motor Drives". IEEE Transactions on Industry Applications, Vol. IA-23, 1987. 